The specimen is now in the Teratological Section of the Museum of the Royal College of Surgeons. In the accompanying photograph the highest pin impales the epiglottis. The metal stylet lies in the trachea and shows the fistula proceeding from the trachea and opening into the stomach. The blind sac of the upper part of the oesophagus is opened and pinned out. The trachea and bronchi are shown opened.

I am indebted to Dr. J. Laurie, medical superintendent, for permission to publish the case, and to $\mathrm{Mr}$. Beggs, clerk of the hospital, who kindly took the photograph.

$$
\text { WaLTER Calvert, D.R.C.O.G. }
$$

Late Obstetrical Officer, Sharoe Green Hospital, Preston now Obstetrical Officer and Deputy Medical Superintendent, Stepping Hill Hospital, Stockport.

Ether Glonus treated by Hexobarbitone Soluble The following case may be considered of sufficient general interest to be recorded.

An obese woman aged 47 was admitted with an acute appendix. Anaesthesia was induced with gas-oxygen-ether given through a standard Boyle apparatus. Induction was uneventful, but just as the surgeon had opened the peritoneum contractions began abruptly and, so far as could be judged, throughout the whole body simultaneously. The anaesthetic was withdrawn at once, and pure oxygen given under as much pressure as was possible with the apparatus being used. The contractions continued with extreme violence and did not permit of a single complete inspiration. Cyanosis rapidly became extreme; $0.5 \mathrm{~g}$. of hexobarbitone soluble (evipan sodium) was dissolved in $5 \mathrm{c.cm}$. of distilled water and administered intravenously. No more than $1 \mathrm{c.cm}$. of the solution had been given when the clonus ceased abruptly. The remainder of the solution was slowly injected and the operation was completed without difficulty. No more anaesthetic was required.

It may be noted that: (1) It is very difficult to enter even a good vein when clonus is present, and almost impossible to be sure of not spilling the solution into the surrounding tissues. (2) Induction required a rather large amount of ether, the appendix was gangrenous, and the theatre unusually hot owing to black-out conditions.

Evesham General Hospital.

H. L. Heath, M.B., Ch.B.

\section{Unusual Cases of Diphtheria}

In view of the recent note by Dr. Manuel Anderson (Journal, July 24, p. 104) on an unusual case of external diphtheritic membrane the following cases seem worthy of record.

CASE I

A boy aged 4 years was admitted to this hospital on Dec. 14, 1940, from the Royal Liverpool Children's Hospital, with large patches of diphtheritic membrane on both tonsils. He had been given 10,000 units of antidiphtheria serum before admission, the throat swab being positive. Shortly before developing the faucial diphtheria he had had a second-stage Edmund's operation for hypospadias.

On admission it was observed that the glans penis was very oedematous and that there was a yellowish membrane covering it blackening in the area of the corona. Cultures taken from this were positive for the Klebs-Loeffler bacillus. A further 40,000 units of A.D.S. was given; the whole penile slough thickened and blackened, and was removed with hydrogen peroxide. The general condition rapidly improved. On Jan. 6, 1941, swabs were taken; the throat proved negative, but the penis positive, as it was again one week later. There were two catgut sutures in the region of the corona; these were removed, and on Jan. 16 a negative swab was obtained.

\section{CASE II}

On Jan. 29, 1941, a woman aged 25, with her child aged 5 weeks, was admitted to hospital. The mother had faucial diphtheria with a large patch of membrane on the left tonsillar area A swab was positive. The course of her case was normal, although in view of her feeding the baby I swabbed the nipples, which were negative for K.L.B.

The child was the interesting case. Before admission there had been a nasal discharge and epistaxis, and the nasal swab was positive for K.L.B. Three weeks previously the child had been circumcised, and there was a filmy membrane on the glans penis and also on the umbilicus, which was still moist. Cultures from both these areas were positive for K.L.B. The throat was negative. 16,000 units of A.D.S. was given. On Jan. 31 there was still some nose-bleeding, but the umbilicus was drying rapidly, forming a blackish scar. The condition of the penis was much the same. On Feb. 3 the black scar had dried and lifted off, leaving a clean healed area. On Feb. 14 small blisters appeared on the penis, but these were negative for K.L.B. and soon cleared up. The mother and child were discharged on March 1, 1941, with swabs from all areas negative.

\section{COMMENT}

As in Dr. Anderson's case, undoubtedly the "raw" areas were ready to be "inoculated" with K.L.B. In these cases trauma was not slight, however; but perhaps the cases bear out the suggestion that trauma plays some part in the introduction of the Klebs-Loeffler bacillus.

I should like to thank Dr. Hillman Gray for access to the records. JohN W. CROWTHER, M.B., Ch.B.

Late Medical Superintendent, Dutton Isolation Hospital.

\section{Reviews}

\section{EXAMINATION OF THE CHEST}

Chest Examination: The Correlation of Physical and $X$-ray Findings in Diseases of the Lung By Richard R. Trail, M.D.P. F.R.C.P. With a
foreword by Sir Walter Langdon-Brown, M.D.P F.R.C.P. (Pp. 107 ; illustrated. 10s. 6d.) London: J. and A. Churchill. 1943.

Wing Cmdr. Trail has written the little book which many of his colleagues have thought of writing but have hitherto shirked the task. 'Its main purpose is to correlate the anatomy and pathology of the lungs with the physical findings-clinical and radiological. The book contains four sections. The first consists of a very useful summary of the static and dynamic anatomy of the lungs, including the normal radiological appearance. The second and main section correlates the abnormal physical and radiological findings in common chest diseases with the pathology. This is well done, and the section contains much information not readily accessible to students. Wing Cmdr. Trail is very dogmatic, as is essential when writing for students, but many of the statements concerning the deductions which can be drawn from physical signs are by no means generally accepted. It is a pity that these were included, because many of the less orthodox claims could have been omitted without loss to the general clarity of his explanations. The two sections containing notes on physical signs and on reading films of abnormal chest conditions bear evidence of their origin in lecture notes, as the author explains in the preface. The former gives many useful hints, but the subsection "Crepitations" will not clear the muddle which exists in many students' minds when classifying adventitious sounds. The section on reading $x$-ray films suffers from its brevity and from insufficient diagrams and reproduction of the conditions described, but internal evidence in the text suggests that this is the fault of the publishers rather than the author

A small volume on these subjects was needed badly, and students will learn much from the careful study of the book. Readers must, however, realize that some of the explanations are useful hypotheses rather than proven facts, and not be discouraged if they fail to obtain as much information is the author does from the physical examination of the patient.

\section{PHYSIOLOGICAL BASIS OF MEDICINE}

The Physiological Basis of Medical Practice. By Charles Herbert Best. M.D., D.Sc., F.R.S., F.R.C.P., and Norman Burke Taylor, M.D. F.R.C.S.Ed. Third edition. (Pp. 1,942; illustrated. 55s.) London:
Bailliere, Tindall and Cox. 1943.

We welcomed the second edition of this valuable book after its appearance in 1939 , since when it has been reprinted three times. Now that a third edition appears we need only repeat our praise, for with one exception the book stands much the same, though there has been revision of detail throughout. The exception is the contribution of Dr. A. M. Wynne on intracellular respiration, which has been entirely rewritten. This is a subject of such growing importance in biochemistry that a brief review of its complexities may be of interest. Intracellular oxidation is primarily concerned with liberation of free energy in the living cell. It is not true, as used to be said, that "all life depends on oxygen," if that statement is read as it stands, for certain organisms and anaerobic bacteria can obtain energy in the complete absence of oxygen by rearranging the atoms in, for example, glucose. In all fermentations there is a cleavage of the molecule, the oxidation of one half being achieved by the reduction of the other half. Even in animal tissues there is an anaerobic mechanism for the liberation of energy. In muscle this comes into play when there is an acute need for energy which the oxygen supply is inadequate to meet. On the other hand, when the oxygen supply is abundant if suppresses the fermentative method. The utilization of intracellular oxygen was studied by Warburg, who revived interest in the muscle pigments detected by McMunn as long ago as 1886 , which were subsequently found to be iron-porphyrin compounds. But Warburg's conception of this as a "respiration enzyme" was inadequate to explain the observed facts. Keilin renamed the pigments cytochromes, of which there are three-a, b, and c. He showed that in whatever way oxidation was effected it involved the loss of one or more electrons, which were regained on reduction. Intracellular 